\title{
Optical and near-infrared follow-up observations of four Fermi/LAT GRBs
}

Sheila McBreen*

School of Physics, University College Dublin, Ireland. E-mail: sheila.mcbreen@ucd.ie

T. Krühler

Max-Planck-Institut für extraterrestrische Physik, 85748 Garching, Germany,

Universe Cluster, Technische Universität München, Boltzmannstrasse 2, D-85748, Germany.

A. Rau, J. Greiner, S. Savaglio, P. Afonso, C. Clemens, R. Filgas, F. Olivares E. , G. P. Szokoly, A. Yoldaş

Max-Planck-Institut für extraterrestrische Physik, 85748 Garching, Germany.

\section{A. Küpcü Yoldaş}

Institute of Astronomy, University of Cambridge, Madingley Road CB3 OHA, Cambridge, UK.

\section{A. Kann, S. Klose, A. Rossi}

Thüringer Landessternwarte Tautenburg, Sternwarte 5, D-07778 Tautenburg, Germany.

\section{A. Updike}

Department of Physics and Astronomy, Clemson University, Clemson, SC 29634, USA.

Fermi can measure the spectral properties of gamma-ray bursts over a very large energy range. Localizations by the instruments on Fermi in combination with follow-up by Swift provide accurate positions for observations at longer wavelengths enabling the determination of redshifts, the true energy budget, host galaxy properties and facilitate comparison with pre-Fermi bursts. Multiwavelength follow-up observations were performed on the afterglows of four bursts with high energy emission detected by Fermi/LAT, GRB 090323, GRB 090328, GRB 090510, GRB 090902B. The observations were obtained in the optical/near-infrared bands with GROND which is mounted at the MPG/ESO $2.2 \mathrm{~m}$ telescope, and additional optical observations of GRB 090323 were obtained with the $2 \mathrm{~m}$ telescope in Tautenburg, Germany. Further observations of GRB 090328, GRB 090510 and GRB 090902B were obtained with the VLT, Chile. Three of the events are classified as long bursts while GRB 090510 is a short GRB.

The afterglows of the long bursts exhibit power-law decay indices $(\alpha)$ from less than 1 to $\sim 2.3$ and spectral indices $\left(\beta_{\text {opt }}\right)$ values from 0.65 to $\sim 1.2$ which are fairly standard for GRB afterglows. Constraints are placed on the jet half opening angles of $\lesssim 2.1^{\circ}$ to $\gtrsim 6.4^{\circ}$, which allows limits to be placed on the beaming corrected energies. These range from $\lesssim 5 \times 10^{50} \mathrm{erg}$ to the one of the highest values ever recorded, $\gtrsim 2.2 \times 10^{52} \mathrm{erg}$ for GRB 090902B. The extremely energetic long Fermi bursts have optical afterglows which lie in the top half of the brightness distribution of all optical afterglows detected in the Swift era or even in the top $5 \%$ if incompleteness is considered.

8th INTEGRAL Workshop "The Restless Gamma-ray Universe"

September 27-30 2010

Dublin Castle, Dublin, Ireland 


\section{Introduction}

The follow-up of gamma-ray bursts (GRBs) detected by the Swift satellite (1) has led to the determination of the distance scale for a large sample of bursts. The Burst Alert Telescope (BAT) (2) is sensitive in the energy range $15-150 \mathrm{keV}$ and has good localization capabilities with typical uncertainties in the arcminute range. Rapid follow-up by Swift's narrow field instruments in the X-rays (XRT) (3) and optical/UV (UVOT)(4) have lead to the arcsecond localizations required for ground-based observers and in turn to spectroscopic redshift measurements of a large sample of GRBs (5) and investigation of their host galaxies (e.g., 6; 7). To date distances to $\sim 200$ GRB sources have been established with redshifts ranging from $z=0.0085(8 ; 9)$ to $z \sim 8.2(10 ; 11)$. However, the BAT has a narrow spectral range and is not able to determine the spectral parameters of the prompt emission of GRBs over a broad energy range. The Fermi Gamma-Ray Space Telescope (12) enables the investigation of the spectrum over seven decades in energy. Fermi hosts two instruments that detect GRBs: the Gamma-ray Burst Monitor (GBM: 13) sensitive to photons from $\sim 8 \mathrm{keV}$ to $\sim 40 \mathrm{MeV}$, and the Large Area Telescope with a spectral coverage from $\sim 30 \mathrm{MeV}$ to $\sim 100 \mathrm{GeV}$ (LAT: 14). Together they provide valuable information on the spectral properties of the prompt emission (e.g., 15; 16;17;18;19), the presence or absence of intrinsic spectral cut-offs (20). GRBs can be localized with uncertainties of degrees by GBM, but arcmin localisations can be provided when the bursts are detected by LAT. In such cases where the best position is provided by LAT, the locations are followed up by Swift/XRT and ground-based observatories, enabling investigation of their afterglows and host properties and facilitating comparison to a general sample.

We report on the optical and near-infrared (NIR) observations of the afterglow of four bursts with high energy emission, GRB 090323, GRB 090328, GRB 090510 and GRB 090902B and compare them to the sample of GRB afterglows to date. Throughout the paper, we adopt concordance $\Lambda \mathrm{CDM}$ cosmology $\left(\Omega_{M}=0.27, \Omega_{\Lambda}=0.73, H_{0}=71 \mathrm{~km} / \mathrm{s} / \mathrm{Mpc}\right)$, and the convention that the flux density of the GRB afterglow can be described as $F_{v}(t) \propto v^{-\beta} t^{-\alpha}$.

\section{Observations}

\section{GRB 090323}

At 00:02:42.63 UT on 23 March 2009, Fermi GBM triggered and located the long burst GRB 090323 (McBreen et al. (2010) and references therein (21)). Swift carried out a Target of Opportunity observation $\sim 19$ hours post burst and a fading afterglow was found. The field of GRB 090323 was observed simultaneously in 7 bands $\left(g^{\prime} r^{\prime} i^{\prime} z^{\prime} J H K_{S}\right)$ by GROND, mounted at the $2.2 \mathrm{~m}$ MPG/ESO telescope at La Silla Observatory, Chile (22) and using the Schmidt-camera at the Alfred-Jensch telescope of the Thüringer Landessternwarte Tautenburg (TLS), Germany. GROND and TLS observations started on 24th March 2009, 27 hours and 45 hours after the GBM trigger, respectively. Observations continued in the following nights and the last GROND epoch was obtained $\sim 100$ days post-trigger. All GROND optical/NIR data presented were reduced and analyzed in standard manner using pyraf/IRAF tasks $(23 ; 24)$.

\section{GRB 090328}

At 09:36:46 UT on 28 March 2009, Fermi GBM triggered and located a long burst GRB 090328

\footnotetext{
* Speaker.
} 
(McBreen et al. (2010) and references therein (21)). An X-ray and bright UV/optical afterglow candidate was found in Swift observations. A spectrum of the optical afterglow was taken with the GMOS instrument mounted on the Gemini South Telescope (25) and the redshift was reported to be $z=0.736$. Optical/NIR follow-up observations by GROND started 1.6 days post trigger. The afterglow was detected in all seven bands consistent with the reported redshift. In addition, GROND imaged the field of GRB 090328 at 2.5, 3.5, 4.5, 6.5 and 11.5 days after the trigger. The host galaxy was detected at a distance of $0.14^{\prime \prime}$ from the afterglow location. Spectroscopy of the afterglow was performed on 2009 March 30.01 UT ( 1.6 days post burst) using the FOcal Reducer and low dispersion Spectrograph 1 (FORS1; 26) at the $8 \mathrm{~m}$ ESO-VLT UT2 telescope (Programme ID: 083.D-0903). The FORS1+2 spectroscopy data were reduced with standard IRAF routines, and spectra were extracted using an optimal (variance-weighted) method.

\section{GRB 090510}

At 00:23:00 UT on 10 May 2009 the Swift BAT triggered on and located the short burst, GRB 090510 and the burst also triggered Fermi/GBM and other instruments (McBreen et al. (2010) and references therein (21)). Swift slewed after 91 seconds and observations with the XRT and UVOT revealed an uncatalogued source (29). Kuin et al. (2009) (30) report that the afterglow of GRB 090510 is detected in almost all UVOT filters which implies that the redshift is less than about 1.5. GROND imaged the field of GRB 090510 starting $6.18 \mathrm{~h}$ after the burst. Observations continued for $4 \mathrm{~h}$ until local twilight and were resumed in the following night for $1.5 \mathrm{~h}$. In the first epoch, the afterglow is detected in the $g^{\prime} r^{\prime} i^{\prime} z^{\prime}$ optical channels, while the $J H K_{S}$ band yield only upper limits and the second epoch only yield upper limits on the afterglow flux. In both epochs a nearby, extended object at a distance of $1.2^{\prime \prime}$ with respect to the afterglow is clearly detected. Given the low spatial separation both objects are blended in the first epoch.

Spectroscopy of the host galaxy of GRB 090510 was performed ( 2.3 days post burst) using FORS2 (26) at the $8 \mathrm{~m}$ ESO-VLT UT1 telescope (Programme ID: 083.D-0903(A)). The preliminary analysis and redshift $z=0.903$ was reported by Rau et al. (2009) (31). Additionally, spectroscopy of a source located $5^{\prime \prime}$ south of the GRB host galaxy was performed $\sim 1.3$ days post burst using FORS2 (300V grism), which yields a redshift consistent with that of the GRB host.

\section{GRB 090902B}

At 11:05:08.31 UT on 2 September 2009 Fermi GBM triggered on a long, bright, hard burst GRB 090902B which was also detected by the LAT (17). A candidate X-ray afterglow within the LAT error circle was reported (27) and subsequently confirmed to be fading (see McBreen et al. (2010) and references therein (21)). The redshift of $z=1.822$ was obtained from an afterglow absorption spectrum by Cucchiara et al. (2009) (28) using the GMOS spectrograph. GROND imaged the field of GRB 090902B starting $13 \mathrm{~h}$ after the Fermi trigger when the position of the GRB was becoming visible satisfying the pointing constraints of the telescope. Observations continued in the following nights by GROND and an additional VLT/FORS2 $R$ band observation was executed at $\sim 23$ days post trigger (ESO DDT Proposal number : 283.D-5059).

\section{Results}

In the standard fireball model, the spectral index, $\beta$, of the afterglow is connected to the temporal index, $\alpha$, via the closure relations (e.g. (32) for a review). These depend on the type of 


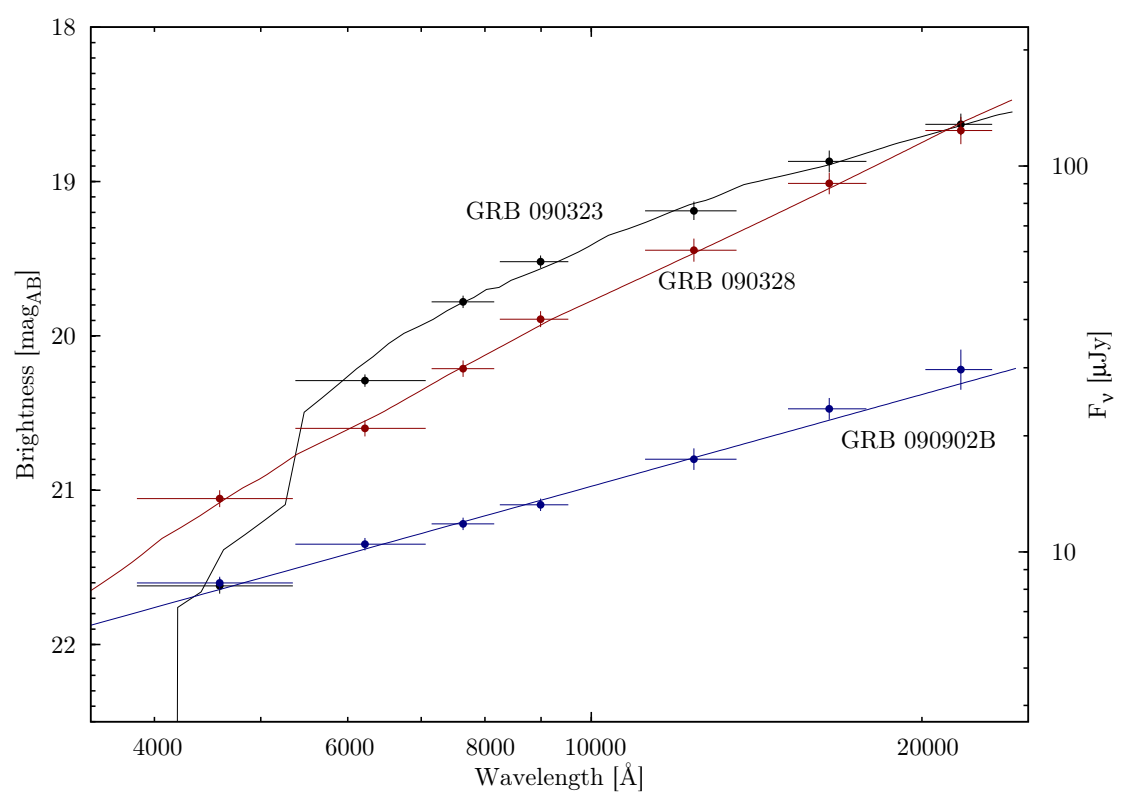

Figure 1: GROND broadband spectral energy distribution of the afterglows of GRB 090323, GRB 090328 and GRB 090902B. Note that the $g^{\prime}$ band points for GRB 090323 and GRB 090902B are overlapping.

circumburst medium, location of the characteristic frequencies in the synchrotron spectrum, and the evolutionary stage of the afterglow (e.g. 33). However, afterglow light curves often display complex temporal behaviour with shallow decays, plateaus, rebrightenings, flares or smooth breaks such that limited sampling strongly affects the inferred light-curve slope (e.g. 34). Hence, we derive the spectral index from the optical to NIR SED, and use the temporal index from the light curve fitting and the closure relations to obtain constraints on the jet properties.

\section{GRB 090323}

The GROND and TLS multicolor light curve of the optical/NIR afterglow starting at $T_{0}+95 \mathrm{ks}$ is well described with a single power-law plus a superimposed bump component and a constant host contribution dominating at later times $\left(\chi^{2}=161 / 138\right.$ d.o.f). The initial power-law decay slope is $\alpha=1.90 \pm 0.01$, with no sign of an additional break at later times. The host galaxy of GRB 090323 was detected in the $r^{\prime}$ and $i^{\prime}$ bands. The obtained spectral index $\beta_{\text {opt }}=0.65 \pm 0.13$ (Fig. 1) is compatible with the observational frequency $v_{\text {opt }}$ being between the typical synchrotron frequency $v_{\mathrm{m}}$ and the cooling frequency $v_{\mathrm{c}}$ for an ISM (density $n=$ constant) or wind $\left(n \propto r^{-2}\right)$ environment. The power-law decay index of the light curve, $\alpha$, is expected to be $\sim 1$ in the pre-jet break, ISM case and is not compatible with the observations. The value of $\alpha$ indicates that the jet break occurred before the start of the observations. However, it is not possible to distinguish between the ISM and wind environments (see, however Cenko et al. 2010 (25)). The post-jet evolution implies that the half opening angle of the jet is smaller than $\theta_{\text {jet }}^{\mathrm{ISM}} \lesssim 2.1^{\circ}$ in an ISM-type circumburst environment (35; 36). The beaming-corrected energy emitted in $\gamma$-rays $E_{\gamma}(1 \mathrm{keV}$ to $10 \mathrm{GeV})$ of GRB 090323 is thus $E_{\gamma} \lesssim 3.3 \times 10^{51} \mathrm{erg}$. In a wind environment (37) the opening angle of the jet would be $\theta_{\text {jet }}^{\text {wind }} \lesssim 1.1^{\circ}$ and the beaming corrected energy would be $E_{\gamma} \lesssim 1.0 \times 10^{51}$ erg $(1 \mathrm{keV}$ to $10 \mathrm{GeV})$.

\section{GRB 090328}

The GROND multicolor light curve of the afterglow of GRB 090328 starting at $\mathrm{T}_{0}+137 \mathrm{ks}$ is 
Table 1: Summary of the burst properties of five Fermi/LAT bursts.

\begin{tabular}{lcccccc}
\hline \hline Burst & Redshift & $\begin{array}{c}E_{\gamma, \text { iso }}{ }^{\mathrm{a}} \\
\text { erg }\end{array}$ & $\alpha$ & $\beta_{\text {opt }}$ & $\begin{array}{c}\theta_{\text {jet }}^{\text {ISM }} \\
{ }^{\circ}\end{array}$ & $\begin{array}{c}E_{\gamma}{ }^{\mathrm{b}} \\
(\text { erg })\end{array}$ \\
\hline \hline & & & & & & \\
GRB 090323 & $3.568 \pm 0.004^{1}$ & $5.1 \times 10^{54}$ & $1.90 \pm 0.01$ & $0.65 \pm 0.13$ & $\lesssim 2.1^{\circ}$ & $\lesssim 3.3 \times 10^{51}$ \\
GRB 090328 & $0.7354 \pm 0.0003^{4}$ & $1.0 \times 10^{53}$ & $2.27 \pm 0.04$ & $1.19_{-0.21}^{+0.24}$ & $\lesssim 5.5^{\circ}$ & $\lesssim 4.6 \times 10^{50}$ \\
GRB 090902B & $1.8229 \pm 0.0004^{2}$ & $3.5 \times 10^{54}$ & $0.94 \pm 0.02$ & $0.79_{-0.19}^{+0.05}$ & $\gtrsim 6.4^{\circ}$ & $\gtrsim 2.2 \times 10^{52}$ \\
GRB 080916C & $4.35^{3}$ & $8.8 \times 10^{54}$ & $1.40 \pm 0.05$ & $0.38_{-0.19}^{+0.20}$ & $\gtrsim 6^{\circ}$ & $\gtrsim 4.8 \times 10^{52}$ \\
GRB 090510 & $0.903 \pm 0.001^{4}$ & $1.1 \times 10^{53}$ & $\ldots$ & $\ldots$ & $\ldots$ & $\ldots$ \\
\hline
\end{tabular}

References: (1) Chornock et al. (2009), (2) Cucchiara et al. (2009), (3) Greiner et al. (2009), (4) McBreen et al. (2010).

Notes: (a) $E_{\gamma \text {,iso }}$ values are quoted in the energy range $1 \mathrm{keV}-10 \mathrm{GeV}$, with the exception of GRB 080916C (10 keV-10 GeV: Abdo et al. 2009a) and GRB $090510(10 \mathrm{keV}-30 \mathrm{GeV}$ : Abdo et al. 2009c). (b) The opening angle and $E_{\gamma}$ are lower in a wind medium for GRB 090323 and 080916C.

remarkably similar to that of GRB 090323. It is well described with a single power-law of index $\alpha=2.27 \pm 0.04$, which is typical of a post-jet break slope plus a superimposed bump component and a constant host contribution dominating at later times $\left(\chi^{2}=35 / 39\right.$ degrees of freedom). The afterglow SED was fitted using a power-law modified by dust reddening as shown in Fig. 1. A pure power-law provides a reasonably good fit to the data with a $\chi^{2}$ of $4.5 / 5$ d.o.f. The resulting power-law index of $\beta_{\mathrm{opt}}=1.46_{-0.08}^{+0.07}$, however, would be surprisingly red if it was intrinsic to the afterglow. In fact, a small amount of extinction of $A_{V}^{\text {host }}=0.22_{-0.18}^{+0.06}$ mag with a SMC type dust attenuation law provides a better fit $\left(\chi^{2}=2.6 / 4\right.$ d.o.f) and a more typical spectral power-law index of $\beta_{\mathrm{opt}}^{\mathrm{SMC}}=1.19_{-0.19}^{+0.21}$. Though statistically not necessarily required, it seems thus very likely that there is mild reddening by dust in the circumburst environment. The LMC and MW dust models cannot be distinguished and return comparable results, with spectral indices of $\beta_{\mathrm{opt}}^{\mathrm{LMC}}=1.07_{-0.12}^{+0.29}$, $\beta_{\mathrm{opt}}^{\mathrm{MW}}=1.16_{-0.21}^{+0.24}$ and $A_{V}^{\text {host }}$ between 0 and $0.4 \mathrm{mag}$.

A spectral index of $\beta_{\mathrm{opt}} \sim 1.2$ is only consistent with temporal index $\alpha=2.27 \pm 0.04$ in a post jet break evolution and the ISM or wind, slow cooling case in the spectral regime above the cooling frequency $v_{\mathrm{opt}}>v_{c}(37 ; 38 ; 39)$. In this case, the electron energy index $p$ is $p=2 \beta=$ $2.4_{-0.4}^{+0.4}$ as obtained from the spectral index. Hence, the observations indicate that the jet break must have occurred before the first GROND observations. This yields an upper limit of $\theta_{\text {jet }}^{\text {ISM }} \lesssim 5.5^{\circ}$ or $\theta_{\text {jet }}^{\text {wind }} \lesssim 4.2^{\circ}(35 ; 36)$, which constrains $E_{\gamma}(1 \mathrm{keV}$ to $10 \mathrm{GeV})$ to $E_{\gamma} \lesssim 4.6 \times 10^{50} \mathrm{erg}$ for an ISM or $E_{\gamma} \lesssim 2.7 \times 10^{50} \mathrm{erg}$ for a wind-type environment.

The spectral and temporal decays of all bursts are presented in Figs 1 and 2, respectively. The indices and energetics were determined for the long GRBs and are presented in Table 1. 


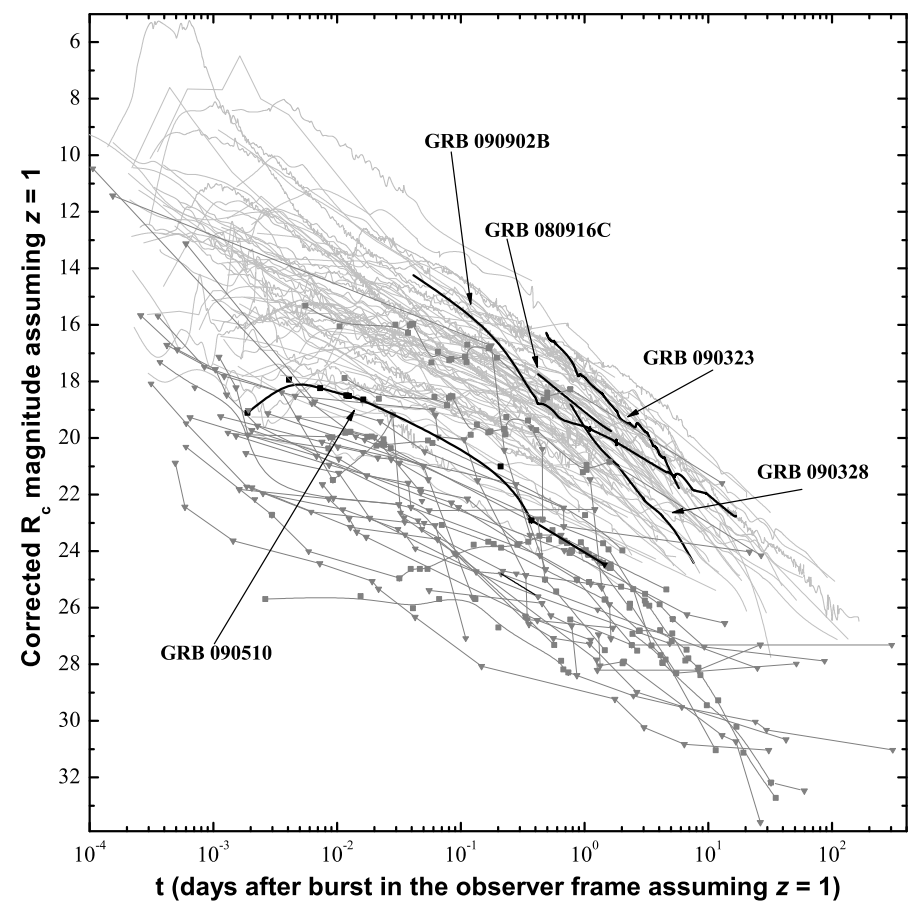

Figure 2: The afterglows of the Fermi/LAT-detected GRBs in comparison with a sample of over 120 well observed afterglows detected until May 2009 (42; 43; 44). All afterglows have been corrected for Galactic extinction and for host-galaxy contribution, where applicable. All afterglows have been additionally corrected for host-galaxy extinction, and have been shifted to a redshift of $z=1$. Long GRBs are shown as light grey lines, whereas short GRBs are thicker grey lines with symbols. Squares are detections, and downward pointing triangles are $3 \sigma$ upper limits.

\section{Summary}

We have presented the optical and NIR follow-up observations of four bursts with high energy emission detected by Fermi/LAT and spectroscopic investigations of two of those events. Since the end of the commissioning phase Fermi/GBM has detected several hundred GRBs, many of which were in the field of view of the LAT instrument. Follow-up of these bursts is of special interest due to the broadband coverage of the prompt emission ( $\mathrm{keV}$ to $\mathrm{GeV}$ ) which allows the spectral properties and energetics of these events to be constrained. It is noteworthy that the redshifts of bursts detected in the $\mathrm{GeV}$ regime by the LAT range from the relatively low-redshift GRB 090328 to the high-redshift of GRB 080916C and that the long bursts have high fluences and very high isotropic gamma-ray energies (although GRB 090328 has a smaller isotropic energy release due to its lower redshift). The properties of these events presented in this paper and another Fermi/LAT event, GRB 080916C, including their afterglows and energetics are summarized in Table 1.

\section{Acknowledgements}

Based on observations made with the ESO Telescopes at the La Silla and Paranal Observatories under programme ID 083.D-0903 and 283.D-5059, the MPG/ESO $2.2 \mathrm{~m}$ Telescope at La Silla Observatory and the Schmidt telescope of the Thüringer Landessternwarte Tautenburg. Part of 
the funding for GROND (both hardware as well as personnel) was generously granted from the Leibniz-Prize to Prof. G. Hasinger (DFG grant HA 1850/28-1). SMB acknowledges support of a European Union Marie Curie Reintegration Grant within the 7th Program under contract number PERG04-GA-2008-239176. TK acknowledges support by the DFG cluster of excellence Origin and Structure of the Universe. S.K. and D.A.K. acknowledge support by DFG K1 766/16-1. DAK acknowledges support by the Thüringer Landessternwarte Tautenburg, and thanks S. Stecklum for observing time, as well as U. Laux and F. Ludwig for performing the observations.

\section{References}

[1] Gehrels, N. et al. 2004, ApJ, 661, 1005

[2] Barthelmy, S. et al. 2004, Space Science Reviews, 120, 143

[3] Burrows, D. et al. 2004, Space Science Reviews, 120, 95

[4] Roming, P. et al. 2004, Space Science Reviews, 120, 165

[5] Fynbo, J. P. U. et al. 2009, ApJSS, 185, 526

[6] Savaglio, S. et al. 2009, ApJ, 691, 182

[7] Perley, D. et al. 2009, AJ, 138, 1690

[8] Tinney, C. et al. 1998, IAUC, 6896, 3

[9] Galama, T. et al. 1998, Nature, 395, 670

[10] Tanvir, N. R. et al. 2009, Nature, 461, 1254

[11] Salvaterra, R. et al. 2009, Nature, 461, 1258

[12] Atwood, W. B et al. 1994, NIMPR, 342, 302

[13] Meegan, C. et al. 2009, ApJ, 702, 791

[14] Atwood, W. B. et al. 2009, ApJ, 697, 1071

[15] Abdo, A. A. et al. 2009, Nature, 462, 331

[16] Abdo, A. A.. et al. 2009, ApJ, 707, 1071

[17] Abdo, A. A. et al. 2009, ApJ, 706, L138

[18] Abdo, A. A. et al. 2009, Science, 323, 1688

[19] Rau, A. et al. 2010, ApJ, 720, 862

[20] Ackermann, M. et al. 2011, ApJ, 729, 114

[21] McBreen, S. et al. 2010, A\&A, 516, A71 
[22] Greiner, J. et al. 2008, PASP, 120, 405

[23] Tody, D. et al. 1993 , in Astronomical Society of the PaciPc Conference Series, Vol. 52, Astronomical Data Analysis Software and Systems II, ed. R. J. Hanisch, R. J. V. Brissenden, \& J. Barnes

[24] Krühler, T. et al. 2008, ApJ, 685, 2824

[25] Cenko, S. B. et al. 2011, ApJ, 732, 29

[26] Appenzeller, I. et al, 1998, The Messenger, 94, 1

[27] Kennea, J. and Stratta, G., 2009, GCN 9868

[28] Cucchiara, A. et al 2009, GCN 9073

[29] Marshall, F. E. and Hoversten, E. A., 2009, GCN 9332

[30] Kuin et al. 2009, GCN 9351

[31] Rau, A. et al, 2009, GCN 9353

[32] Piran, T., 2004, Reviews of Modern Physics, 76, 1143

[33] Racusin, J. L., 2009, ApJ, 698, 43

[34] Greiner, J, 2009, ApJ, 693, 1912

[35] Sari, R. et al 1999, ApJ, 519, L17

[36] Frail, J, 2001, ApJ, 562, L55

[37] Chevalier, R. A. and Li, Z.-Y., 2000, ApJ, 562, 195

[38] Sari, R., Piran, T. and Narayan, R., 1998, ApJ, 487, L17

[39] Zhang, B. and Mészáros, P., 2004, International Journal of Modern Physics A, 19, 2385

[40] Bertin, E. and Arnouts, S, M. et al. 1996, A\&AS, 117, 393

[41] Hamuy, M. et al. 1992, ApJ, 104, 533

[42] Kann, D. A et al. 2006, ApJ, 641, 993

[43] Kann, D. A. et al. 2010, ApJ, 720, 1513

[44] Kann, D. A. et al. 2011, ApJ, in press (arXiv:0804.1959v2)

[45] Olofsson, G. et al 2009, GCN 9338

[46] Amati, L. et al 2008, MNRAS, 391, 577

[47] Ghirlanda, G. et al 2007, A\&A, 466, 127

[48] Chornock, R. et al 2009, GCN 9028

[49] Greiner, J. et al 2009, A\&A, 498, 89 